\author{
ANNALES \\ POLONICI MATHEMATICI \\ XLII (1983)
}

\title{
Inégalités différentielles fonctionnelles du type elliptique
}

\author{
par Marian Malec (Cracovie)
}

Dédié à la mémoire de Jacek Szarski

Résumé. On démontre, sous certaines conditions, que les théorèmes sur les inégalités différentielles fonctionnelles fortes et faibles sont valables. Dans la dernière partie de la note on a donné un principe du maximum ainsi qu'un théorème sur l'unicité des solutions du système (4.3) avec des conditions aux limites de la forme (4.4).

1. On connaît bien le principe du maximum, ainsi que le théorème sur l'unicité de la solution du problème aux limites pour l'équation non linéaire différentielle du type elliptique de la forme

$$
f\left(x, u(x), u_{x}(x), u_{x x}(x)\right)=0
$$

où $u: R^{m} \supset G \rightarrow R^{1}, u_{x}=\operatorname{grad} u, u_{x x}$ est la matrice des secondes dérivées par rapport à $\left(x_{1}, \ldots, x_{n}\right)$ (cf. par exemple [2]).

Ces théorèmes bien connus constituent des conséquences de théorèmes très généraux sur les inégalités différentielles du type elliptique. Nous allons établir des théorèmes sur les inégalités différentielles fonctionnelles fortes et faibles du type elliptique.

Nous allons formuler des théorèmes sur les inégalités différentielles fonctionnelles sans d'ailleurs insister sur les hypothèses les plus générales possible. Ci-dessous nous présentons des résultats qui sont dans un certain sens une continuation des résultats que j'ai obtenus plus tôt dans [1].

2. Soit $D \subset R^{n}$, où $R^{n}$ désigne l'espace euclidien réel à $n$ dimensions. Le symbole $D_{e}$ désignera l'ensemble de tous les points $\bar{x} \in \bar{D}$ pour lesquels il existe un nombre réel $\delta=\delta(x)>0$ tel que

$$
\left\{x \in R^{n} \backslash\{\tilde{x}\}: \prod_{i=1}^{n} \sum_{\substack{j=1 \\ j \neq i}}\left(x_{j}-\tilde{x}_{j}\right)^{2}=0, \sum_{i=1}^{n}\left(x_{i}-\tilde{x}_{i}\right)^{2}<\delta^{2}\right\} \subset \operatorname{Int} D
$$

où $\bar{D}$ dénote la fermeture de l'ensemble $D$ et $\operatorname{Int} D$ son intérieur. 
Il est évident que Int $D \subset D_{e} \subset \bar{D}$.

L'ensemble $\partial_{e} D=\bar{D} \backslash D_{e}$ sera dit frontière elliptique de l'ensemble $D$.

Soit $\partial_{e} D=\Gamma \cup \Sigma$ ( $\Gamma$ et $\Sigma$ peuvent avoir des points communs et l'un des ensembles $\Gamma$ et $\Sigma$ peut être vide). On suppose qu'à chaque point $\bar{x} \in \Sigma$ correspond une seule suite $n(\bar{x})=\left\{x_{k}(\bar{x})\right\} \subset D_{e}(k=1,2, \ldots)$ telle que

$$
\lim _{k \rightarrow \infty} x_{k}(\bar{x})=\bar{x} \text {. }
$$

Soit $\omega: D_{e} \cup\{\bar{x}\} \rightarrow R^{1}$ où $\bar{x} \in \Sigma$. Nous définissons une fonction $\partial \omega / \partial n$ de la manière suivante:

$$
\frac{\partial \omega}{\partial n}(\bar{x})=\limsup _{k \rightarrow \infty} \frac{\omega\left(x_{k}(\bar{x})\right)-\omega(\bar{x})}{\left|x_{k}(\bar{x})-\bar{x}\right|}
$$

où $|x|=\sqrt{x_{1}^{2}+\ldots+x_{n}^{2}}$ pour $x \in R^{n}$.

Si $\varphi: D_{e} \rightarrow R^{m}$ et $\psi: D_{e} \rightarrow R^{m}$, l'écriture " $\varphi \leqslant \psi$ dans $\Gamma_{\infty}$ " exprime le fait que pour toute la suite $\left\{x_{k}\right\} \subset D_{e}(k=1,2, \ldots)$ telle que $\left|x_{k}\right|$ $\rightarrow \infty$, on a

$$
\limsup _{k \rightarrow \infty}\left[\varphi^{i}\left(x_{k}\right)-\psi^{i}\left(x_{k}\right)\right] \leqslant 0
$$

pour $i=1, \ldots, m$.

Dans ce qui suit on suppose que $\Sigma=\Sigma_{1} \cup \ldots \cup \Sigma_{m}$ et $\Gamma_{i}=\partial_{e} D \backslash \Sigma_{i}$ $(i=1, \ldots, m)$. L'écriture " $\varphi \leqslant \psi$ dans $\Gamma_{+}$" signifie que pour tout la suite $\left\{x_{k}\right\} \subset D_{e}(k=1,2, \ldots)$ telle que $\lim x_{k} \in \Gamma_{i}$, on a l'inégalité (2.4).

Une application $u: R^{n} \supset A \rightarrow \stackrel{\substack{k \rightarrow \infty \\ \rightarrow}}{n}$ sera dite application de classe $C^{k}$ dans $A$ (nous écrirons alors $u \in C^{k}(A)$ ) lorsqu'il existe un prolongement de $u$ sur un ensemble ouvert $G \supset A$ tel qu'il est de classe $C^{k}$ dans $G$.

LEMME. Soit $h^{i}: D_{\bullet} \cup \Sigma_{i} \rightarrow R^{1}, h^{i} \in C^{0}\left(D_{e} \cup \Sigma_{i}\right)(i=1, \ldots, m)$ et supposons que

(a) $h \geqslant 0$ dans $\Gamma_{+}$et dans $\Gamma_{\infty}$,

(b) $\frac{\partial h^{i}}{\partial n}(\bar{x})<0$ en chaque point $\bar{x} \in \Sigma_{i}$ pour lequel $h^{i}(\bar{x}) \leqslant 0 \quad(<0)$, $i=1, \ldots, m$,

(c) il existe un indice $j(1 \leqslant j \leqslant m)$ et $\tilde{x} \in D_{e} \cup \Sigma_{j}$ tels que $h^{j}(\tilde{x}) \leqslant 0$ $(<0)$.

Alors il existe un indice $k(1 \leqslant k \leqslant m)$ et un point $a \in D_{e}$ tels que

$$
\min _{1<i<m} \inf _{x \in D_{e} \cup \Sigma_{i}} h^{i}(x)=h^{k}(a) \text {. }
$$

Démonstration. Démontrons d'abord qu'il existe un point $a_{j} \in D_{e}$ tel que

$$
\inf _{x \in D_{e} \cup \Sigma_{j}} h^{j}(x)=h^{j}\left(a_{j}\right)
$$


Remarquons qu'à cause de la définition (2.3) et des hypothèses (b), (c), nous obtenons

$$
\inf _{x \in D_{e} \cup \Sigma_{j}} h^{j}(x)=\inf _{x \in D_{e}} h^{j}(x)
$$

(voir aussi (2.2)). Soit

$$
A(\tilde{x})=\left\{x \in D_{e} \cup \Sigma_{j}: h^{j}(x)<h^{j}(\tilde{x})\right\} .
$$

Si $A(\tilde{x})=\emptyset$, alors $\tilde{x} \in D_{e}$ (cf. (b) et (2.3)) et en posent $a_{j}=\tilde{x}$, on obtient (2.6).

Supposons maintenant que $A(\tilde{x}) \neq \emptyset$ et soit $x_{1} \in A(\tilde{x})$. Alors

$$
h^{j}\left(x_{1}\right)<h^{j}(\tilde{x}) \leqslant 0 .
$$

Si $A\left(x_{1}\right)=\varnothing$, alors $x_{1} \in D_{e}$ et en mettant $a_{j}=x_{1}$, on a (2.6). Soit maintenant $A\left(x_{1}\right) \neq \emptyset$. Alors l'ensemble $\overline{A\left(x_{1}\right)}$ est borné puisque $h^{j} \geqslant 0$ dans $\Gamma_{\infty}$ (hypothèse (a)). L'ensemble $\overline{A\left(x_{1}\right)}$ est donc un compact dont la frontière $\partial A\left(x_{1}\right)$ est contenue dans $D_{e} \cup \Sigma_{j}$ puisque $h^{j} \geqslant 0$ dans $\left(\Gamma_{j}\right)_{+}$ (hypothèse (a)). Donc

$$
\inf _{x \in D_{e} \cup \Sigma_{j}} h^{j}(x)=\min _{x \in \overline{\Delta\left(x_{1}\right)}} h^{j}(x)=h^{j}\left(a_{j}\right)
$$

où $a_{j} \in D_{e} \cup \Sigma_{j}$. Compte tenu de l'hypothèse (b) et de la définition de l'ensemble $A\left(x_{1}\right)$ ainsi que de $(2.7),(2.3)$, nous constatons que $a_{j} \notin \Sigma_{j}$, d' où l'égalité (2.6).

Soit maintenant $j_{1}, \ldots, j_{s}\left(1 \leqslant j_{k} \leqslant m ; l_{k}=1, \ldots, s\right)$ l'ensemble des indices pour lesquels il existe un point $\tilde{x}_{j_{k}} \in D_{e} \cup \Sigma_{j_{k}}$ tel que $h^{j_{k}}\left(x_{j_{k}}\right) \leqslant 0$ $(k=1, \ldots, s)$. Il résulte de là et du raisonnement précédent (voir $(2.6))$ qu'il existe un point $a \in D_{e}$ en lequel l'égalité (2.5) est satisfaite, ce qui termine la démonstration du lemme.

Une application

$$
w=\left(w^{1}, \ldots, w^{m}\right): D_{0} \ni x \rightarrow\left(w^{1}(x), \ldots, w^{m}(x)\right) \in R^{m}
$$

où $D_{0} \supset \bar{D}$ sera dite $\Sigma$-régulière en $D_{e}$ si $w^{i} \in C^{0}\left(D_{e} \cup \Sigma_{i}\right) \cap C^{2}\left(D_{e}\right)$ pour $i=1, \ldots, m$.

HYPOTHĖSE H.

$\left(\mathrm{H}_{1}\right)$ Les fonctions réelles $f^{i}(x, z, q, r, w)(i=1, \ldots, m)$ sont définies pour $x \in D_{e}, z \in R^{m}, q \in R^{n}, r \in R^{n \times n}, w \in Y$ où $Y$ est un sous-ensemble fixé (pas nécessairement sous-espace linéaire) de l'ensemble des applications $\Sigma$-régultières dans $D_{e} ; f^{i}$ est non croissante par rapport d̀ $w(i=1, \ldots, m)$. on arrive $\grave{a}$

$\underset{\left(\mathrm{H}_{2}\right)}{\left(\mathrm{Si}_{i} i s \in R^{m}, \underset{1 \leqslant i \leqslant m}{\max } s^{i}\right.}=s^{j} \geqslant 0, s(j)=(\underbrace{s^{j}, \ldots, s^{j}}_{m \text { fois }}), w \in Y, w+s(j) \in Y$, pour $x \in D_{e}, z \in R^{m}, q \in R^{n}, r \in R^{n \times n}$. 
$\left(\mathrm{H}_{3}\right)$ La fonction réelle $g^{i}(x, p)$ est définie pour $x \in \Sigma_{i}, p \in R^{1}$ et non croissante par rapport à $p$.

Remarque 1. L'hypothèse $\left(\mathrm{H}_{2}\right)$ est satisfaite, par exemple, lorsque la fonction $w^{i}$ est intégrable au sens de Riemann sur le compact $K_{i} \subset R^{n}$ $(i=1, \ldots, m)$;

$$
f^{f}(x, z, q, r, w)=\sum_{i=1}^{m}\left[L_{i j} z^{i}-\int_{K_{i}} w^{i}(x) d x\right],
$$

où $L_{i j} \in R^{\mathbf{1}}, L_{i j} \leqslant 0$ pour $i \neq j$ et

$$
\sum_{i=1}^{m}\left(L_{i j}-\left|\boldsymbol{K}_{i}\right|\right) \geqslant 0
$$

où $\left|K_{i}\right|$ dénote la mesure de l'ensemble $K_{i}(i=1, \ldots, m)$. Dans le cas où $m=1$ et $Y=\emptyset$ l'hypothèse $\left(\mathrm{H}_{2}\right)$ est équivalente à la croissance faible de $f^{1}$ par rapport à la variable $z \in R^{1}$.

Dans suite, on désigne pour $x \in D_{e}$ l'ensemble suivant

$$
H(x)=\left\{h \in R^{n}: \exists \alpha=a(x, h)>0 \quad \forall \beta \in[0, \alpha), \beta \cdot h \in D_{e}\right\}
$$

et on définit la classe de matrices:

$$
R(x)=\left\{r \in R^{n \times n}: \forall h \in H(x) \sum_{j, k=1}^{n} r_{j k} h_{j} h_{k} \geqslant 0\right\} .
$$

Remarque 2. Il résulte de la définition de l'ensemble $D_{e}$ (voir (2.1)) que si $x \in D_{e}$, alors $H(x) \neq \varnothing$ et si $x \in \operatorname{Int} D \subset D_{e}$, alors $H(x)=R^{n}$ et $R(x)$ est une classe de matrices semi-définie positivement.

Remarque 3. Si $d: D_{e} \rightarrow R^{1}, d \in C^{2}\left(D_{e}\right)$ et $\inf _{x \in D} d(x)=d(a), a \in D_{e}$, on a $d_{x}(a)=(\operatorname{grad} d)(a)=0$ et $d_{x x}(a)=\left(d_{x_{j} x_{k}}(a)\right)_{j, k=1}^{n} \in R(a)$. Ce fait découle directement de la formule de Taylor et, e'est pourquoi nous en omettons la démonstration.

DÉFINITION. On appelle l'application $v \in Y$ elliptique à gauche par rapport à $f$ dans l'ensemble $D_{e}$, si

$$
\begin{aligned}
r \in R(x) \Rightarrow f^{i}\left(x, v(x), v_{x}^{i}(x), v_{x x}^{i}(x)-r, v\right) & \\
& \geqslant f^{i}\left(x, v(x), v_{x}^{i}(x), v_{x x}^{i}(x), v\right)
\end{aligned}
$$

pour $x \in D_{e}$ et $i=1, \ldots, m$ où $v_{x}^{i}(x)=\left(\operatorname{grad} v^{i}\right)(x), v_{x x}^{i}(x)=\left(v_{x_{j} x_{k}}^{i}(x)\right)_{j, k=1}^{n}$.

3. ThÉoRìme 1 (sur les inégalités fortes). On suppose que

$1^{\circ} u, v+s \in Y$ pour toute application constante $s \geqslant 0$;

$2^{\circ}$ les hypothèses $\mathrm{H}$ sont satisfaites;

$3^{\circ} v$ est une application elliptique à gauche par rapport à $f$ dans l'ensemble $D_{e}$; 
$4^{\text {o }} u^{i} \leqslant v^{i}$ sur $\left(D_{0} \backslash \bar{D}\right) \cup \Gamma_{i}(i=1, \ldots, m)$;

$5^{\circ} u \leqslant v$ dans $\Gamma_{+}$et dans $\Gamma_{\infty}$;

$6^{\circ}-\frac{\partial u^{i}}{\partial n}(\bar{x})+g^{i}\left(\bar{x}, u^{i}(\bar{x})\right)<-\frac{\partial v^{i}}{\partial n}(\bar{x})+g^{i}\left(\bar{x}, v^{i}(\bar{x})\right)$ pour les points $\bar{x} \in \Sigma_{i}$ en lesquels $v^{i}(\bar{x}) \leqslant u^{i}(\bar{x}) \quad(i=1, \ldots, m)$;

$7^{\circ} f^{i}\left(x, u(x), u_{x}^{i}(x), u_{x x}^{i}(x), u\right)<f^{i}\left(x, v(x), v_{x}^{i}(x), v_{x x}^{i}(x), v\right)$ pour $x \in D_{e}$ et $i=1, \ldots, m$.

Sous ces hypothèses, on a

$$
u^{i}<v^{i} \quad \text { sur } \quad D_{e} \cup \Sigma_{i}(i=1, \ldots, m) .
$$

Démonstration. Soit $d=v-u$ et supposons que (3.1) n'ait pas lieu, ce qui signifie qu'il existe un indice $j(1 \leqslant j \leqslant m)$ ainsi qu'un point $\tilde{x} \in D_{e} \cup \Sigma_{j}$ tels que $d^{j}(\tilde{x}) \leqslant 0$. Compte tenu du lemme (voir les hypothèses $5^{\circ}, 6^{\circ}$ et $\left.\left(\mathrm{H}_{3}\right)\right)$ on obtient

$$
\min _{1 \leqslant i \leqslant m}\left[\inf _{x \in D_{e} \cup \Sigma_{i}} d^{i}(x)\right]=d^{k}(a) \leqslant 0
$$

où $a \in D_{e}$. П résulte de (3.2) et de l'hypothèse $4^{\circ}$ que

$$
d^{i}(x) \geqslant d^{k}(a) \quad \text { pour } x \in D_{0}, i=1, \ldots, m
$$

ce qui conduit, en vertu de la définition de l'application $d$, à

$$
u \leqslant v+s(k) \quad \text { pour } x \in D_{0}
$$

où $s(k)=-(\underbrace{d^{k}(a), \ldots, d^{k}(a)}_{m \text { iois }}) \geqslant 0$.

$\Pi$ est également à remarquer que si $s=-\left(d^{1}(a), \ldots, d^{m}(a)\right)$, alors $\max _{1<i<m} s^{i}=s^{k} \geqslant 0$.

Toutes les considérations précédentes nous permettent d'utiliser l'inégalité (2.8). En tenant compte du fait que la fonction $f^{t}$ est monotone par rapport à la dernière variable ainsi que de l'hypothèse $3^{\circ}$ (voir aussi la remarque 3$)$, on a

$$
\begin{aligned}
& f^{k}\left(a, u(a), u_{x}^{k}(a), u_{x x}^{k}(a), u\right)-f^{k}\left(a, v(a), v_{x}^{k}(a), v_{x x}^{k}(a), v\right) \\
& \geqslant f^{k}\left(a, u(a), u_{x}^{k}(a), u_{x x}^{k}(a), v+s(k)\right)-f^{k}\left(a, v(a), v_{x}^{k}(a), v_{x x}^{k}(a), v\right) \\
& =f^{k}\left(a, v(a)+u(a)-v(a), v_{x}^{k}(a), v_{x x}^{k}(a)-\left(v_{x x}^{k}(a)-u_{x x}^{k}(a)\right), v+s(k)\right)- \\
& \quad-f^{k}\left(a, v(a), v_{x}^{k}(a), v_{x x}^{k}(a), v\right) \geqslant 0 .
\end{aligned}
$$

Mais cela est en contradiction avec l'hypothèse $7^{\circ}$.

La démonstration du théorème 1 est ainsi terminée.

Remarque 4. On peut aussi affaiblir effectivement l'hypothèse $\left(\mathrm{H}_{2}\right)$ en la remplaçant par la condition: 


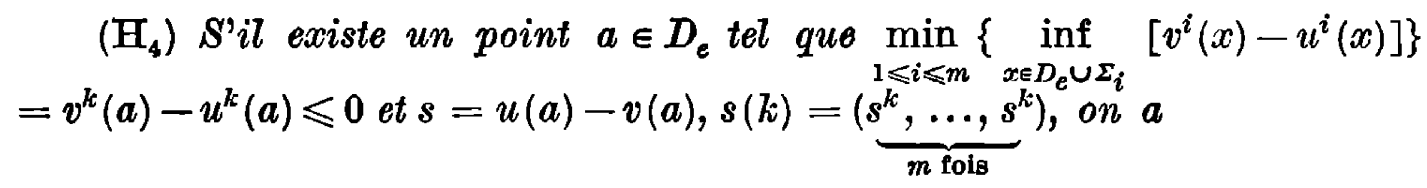

$$
\begin{aligned}
f^{k}\left(a, v(a)+s, v_{x}^{k}(a), v_{x x}^{k}(a)-r,\right. & +s(k))- \\
& -f^{k}\left(a, v(a), v_{x}^{k}(a), v_{x x}^{k}(a), v\right) \geqslant 0
\end{aligned}
$$

pour chaque matrice $r \in R(a)$ (voir (2.10)).

Alors, sous les hypothèses $1^{\circ},\left(\mathrm{H}_{1}\right),\left(\mathrm{H}_{4}\right),\left(\mathrm{H}_{3}\right), 4^{\circ}, 5^{\circ}, 6^{\circ}$ et $7^{\circ}$ a lieu l'inégalité (3.1). La démonstration de ce fait est presque identique à celle $\mathrm{du}$ théorème 1 .

$\eta$ est cependant à noter que l'hypothèse $\left(\mathrm{H}_{4}\right)$ est difficile à vérifier dans les cas concrets.

4. Thॄ́onème 2 (sur les inégalités faibles). Si les hypothèses $1^{\circ},\left(\mathbf{H}_{1}\right)$, $\left(\mathrm{H}_{3}\right), 3^{\circ}, 4^{\circ}, 5^{\circ}\left(6^{\circ}>\right)$ ainsi que l'hypothèse $\left(\mathrm{H}_{2}>\right)$, obtenues à partir de $6^{\circ}$ et de $\left(\mathrm{H}_{2}\right)$ par en y remplaçant les inégalités faibles des inégalités fortes, sont satisfaites ot si

$$
\begin{aligned}
f^{i}\left(x, u(x), u_{x}^{i}(x), u_{x x}^{i}(x), u\right) \leqslant f^{i}(x, v(x), & \left.v_{x}^{i}(x), v_{x x}^{i}(x), v\right) \\
& \left(x \in D_{e}, i=1, \ldots, m\right),
\end{aligned}
$$

on $a$

$$
u \leqslant v \text { sur } D_{0} .
$$

La démonstration du théorème 2 est tout à fait pareille à celle $d u$ théorème 1, c'est pourquoi nous l'omettons.

En ce qui concerne le théorème 2 on peut formuler une remarque analogue à la remarque 4.

En tant que conclusions classiques découlant du théorème 2 nous obtenons les théorèmes énoncés ci-dessous.

Univocité de la solution du problème aux limites. Sous les hypothèses $\left(\mathbf{H}_{1}\right)$ et $\left(\mathrm{H}_{2}>\right)$ introduites précédemment sur la fonction $f^{i}$, le système d'équations différentielles fonctionnelles

$$
f^{i}\left(x, u(x), u_{x}^{i}(x), u_{x x}^{i}(x), u\right)=0 \quad\left(i=1, \ldots, m, x \in D_{e}\right)
$$

possède tout au plus une solution $\Sigma$-régulière et elliptique à gauche par rapport à $f$ dans l'ensemble $D_{e}$ et satisfaisant aux conditions aux limites

$$
\begin{aligned}
& u^{i}=\varphi^{i} \operatorname{sur}\left(D_{0} \backslash \bar{D}\right) \cup \Gamma_{i} ; \quad u=\psi \text { dans } \Gamma_{+} \text {et dans } \Gamma_{\infty} ; \\
& \frac{\partial u^{i}}{\partial n}(\bar{x})=g^{i}\left(\bar{x}, u^{i}(\bar{x})\right) \quad \text { pour } \bar{x} \in \Sigma_{i}(i=1, \ldots, m)
\end{aligned}
$$

où $g^{i}(x, p)\left(x \in \Sigma_{i}, p \in R^{1}, i=1, \ldots, m\right)$ est fortement décroissante par rapport à $p$. 
Principe du maximum. Sous les hypothèses précédentes supposons que pour $A=\left(A^{1}, \ldots, A^{m}\right)=$ const on ait

(4.5) $\quad f^{i}(x, A, 0,-r, A) \geqslant f^{i}(x, A, 0,0, A) \geqslant 0, \quad g^{i}\left(\bar{x}, A^{i}\right) \geqslant 0$

pour $i=1, \ldots, m, x \in D_{e}, r \in R(x), \bar{x} \in \Sigma_{i}$.

Alors, pour la solution $\Sigma$-régulière en $D_{e}$ du système (4.3) satisfaisant aux inégalités aux limites

$$
\begin{aligned}
u^{i} \leqslant A^{i} \text { sur } & \left(D_{0} \backslash \bar{D}\right) \cup \Gamma_{i} ; \quad u \leqslant A \text { dans } \Gamma_{+} \text {et dans } \Gamma_{\infty} ; \\
& -\frac{\partial u^{i}}{\partial n}(\bar{x}) \geqslant g^{i}\left(\bar{x}, u^{i}(\bar{x})\right) \text { pour } \bar{x} \in \Sigma_{i} \text { et } i=1, \ldots, m,
\end{aligned}
$$

on a $u(x) \leqslant A$ sur $D_{0}$.

\section{Travaux cités}

[1] M. Malec, Un analogue du lemme Nagumo-Westphal pour des équations différentielles fonctionnelles du type elliptique, à paraître dans Bull. Acad. Polon. Sci., Sér. Sci. Math. Astronom. Phys.

[2] W. Walter, Differential and integral inequalities, Springer-Verlag, BerlinHeidelberg-New York 1970. 